\title{
Psychometric assessments of life quality and voice for teachers within the municipal system, in Bauru, SP, Brazil
}

\author{
Janaína Gheissa MARTINELLO'1, José Roberto Pereira LAURIS², Alcione Ghedini BRASOLOTTO³
}

\begin{abstract}
1- Speech-Language Pathologist, MSc in Speech-Language Pathology, Department of Speech-Language Pathology and Audiology, Bauru School of Dentistry, University of São Paulo, Bauru, SP, Brazil.

2- PhD, Associate Professor, Department of Pediatric Dentistry, Orthodontics and Community Health, Bauru School of Dentistry, University of São Paulo, Bauru, SP, Brazil.

3- MSc, PhD, Assistant Professor, Speech-Language Pathologist, Department of Speech-Language Pathology and Audiology, Bauru School of Dentistry, University of São Paulo, Bauru, SP, Brazil.
\end{abstract}

Corresponding address: Profa. Dra. Alcione Ghedini Brasolotto - Faculdade de Odontologia de Bauru - USP - Departamento de Fonoaudiologia - Alameda Dr. Octávio Pinheiro Brisolla 9-75 - C.P. 73 - 17012-901 - Bauru - SP - Brasil - Phone: +55 1432358332 - e-mail: alcione@usp.br

Received: July 25, 2010 - Accepted: August 16, 2011

\section{ABSTRACT}

Studies show a high prevalence of vocal alterations among teachers. One of the criteria $\checkmark$ for the establishment of the prevalence of vocal alteration is based on teachers' selfperception. Objective: This study aimed at comparing voice-disordered quality of life measures between a group of teachers who reported vocal alteration and a group of teachers who did not, by verifying the teachers' perception regarding the impact of vocal alteration in the different dimensions of voice quality of life. Material and Methods: Ninety-seven (97) teachers answered three psychometric protocols of voice quality of life: Voice Handicap Index (VHI), Voice-Related Quality of Life (V-RQOL), and the Voice Activity Participation Profile (VAPP), in addition to a questionnaire for characterization of the sample. Results: The results were that $39.8 \%$ of the teachers reported vocal alteration. When comparing voice measures between the groups (with and without vocal alteration), statistically significant differences were observed: the total score of VHI, total score of V-RQOL and total score of VAAP and its dimensions. It was also verified that the physical dimension of VHI has a greater impact among the dimensions of this protocol. For VRQOL, the most striking dimension was the physical functioning domain, both indicating the laryngeal discomfort and the impact of voice on communication, in teachers with and without complaints. As for VAAP, no domain prevailed over the others in the group with no complaints. For teachers with complaints, three domains, i.e., daily communication, work, and emotions have a greater impact than social communication. The limitation and restriction scores were calculated as well, and it was observed the limitation of activities is greater than the restriction of activities, both in the group with and the group without complaints. Conclusion: One may conclude that the teachers who reported vocal alterations better realize the impact of voice in different dimensions of voice quality of life.

Key words: Voice. Teachers. Quality of life.

\section{INTRODUCTION}

Among voice professionals, teachers are the ones presenting the highest risks of vocal alterations ${ }^{14,15,18,21}$. Physical factors such as inadequate space, number of students per classroom, and psycho-emotional factors such as professional adaptation problems and stress, may contribute to the development of vocal alterations ${ }^{3,4,17}$, which might limit or restrict their professional performance, rendering their participation in the teaching profession nonviable ${ }^{14,15,22}$. Thus, it is necessary to investigate whether a vocal alteration may bring negative consequences to the life quality (LQ) of the teacher.

Health professionals utilize psychometric 
questionnaires that are thoroughly elaborated and idealized according to the declarations of the patients, to assess, objectively, the results of treatment or life quality ${ }^{6}$. On the life quality assessment, the impact of the disease is evaluated based on the perception of the patient and not on the view of the health professional. The multidimensionality of human beings and their perceptions (subjectivity) are the common points of LQ assessments in the field of health ${ }^{5,16}$.

The research aimed at investigating and relating vocal health to life quality contributes to the comprehension of the subjects: how individuals perceive their vocal health, and the impact of voice in different dimensions of human existence, e.g., physical, emotional, social, etc.

Hence, the knowledge of how teachers perceive a vocal alteration, and its impact on their $L Q$, helps health professionals and those responsible for health promotion policies develop more effective programs to guarantee the health of teachers.

This study aimed at verifying the self-perception of teachers regarding the impact of vocal use on their LQ and development of their daily activities, by establishing the vocal alteration prevalence reported by the teachers and comparing LQ and voice measurements between two groups of teachers, i.e., the group that reported vocal alteration and the one reporting no vocal alteration.

\section{MATERIAL AND METHODS}

Teachers who affirmatively answered the question: "Have you had any alteration in your voice?"4, comprised the group of teachers who reported voice alteration. Data were collected on the symptomatology and sensation of vocal alteration in the teachers studied ${ }^{4}$, both in those who reported vocal alteration and in those who did not

Ninety-seven (97) teachers with the public system in Bauru, SP, Brazil, responded to three questionnaires that measured their LQ: the Vocal Handicap Index (VHI), the Life Quality and Voice protocol (V-RQOL) and the Profile of Participation and Vocal Activity (VAPP), validated to Portuguese ${ }^{2,6}$.

The VHI and V-RQOL protocols utilize five-point scales. VHI assesses the organic, functional, and emotional multi-dimensions to establish a vocal disadvantage index. The greater the score, the greater the vocal disadvantage identified by the questionnaire. On the other hand, V-RQOL assesses voice $L Q$, based on the dimensions of physical function and social-emotional domain. The greater the $\mathrm{V}$-RQOL score, the better the relationship between voice and LQ.

The VAPP protocol utilizes a $10 \mathrm{~cm}$ analogicalvisual scale in each question. The instrument proposes the analysis of sections: voice problem severity level, voice effect at work, in daily communication, in social communication, and in emotion. The greater the score, the greater the impact of voice on the performance of daily activities.

The Mann-Whitney test was utilized to compare the group that reported vocal alteration (Group II) and the group that reported no vocal alteration (Group I). The Friedman test was used for comparison between the sub-areas of VHI and VAPP, and the Dunn test was utilized for multiple comparisons. For comparison between the domains of $\mathrm{V}$-RQOL and restriction and limitation of VAPP, the Wilcoxon test was utilized for repeated measurements. A 5\% significance level was adopted in all statistical tests.

This study was authorized by the Education Bureau of Bauru and by the Research Ethics Committee of the Bauru School of Dentistry - USP, no $169 / 2007$.

\section{RESULTS}

Out of the 97 teachers studied, $92.9 \%$ were females and $7.1 \%$ males. The prevalence of teachers who reported vocal alteration (GII) at the time of the research was $39.8 \%$ and the prevalence among the teachers who reported no vocal alteration was $60.2 \%$ (GI).

Among the teachers who denied vocal alteration (GI), 19\% reported they sometimes are hoarse and $13.6 \%$ of the teachers reported both lack of air and voice failure. Vocal sensations, such as difficulty speaking, were reported by $33.9 \%$ of the teachers, followed by effort to speak (44.1\%), burning (25.4\%), and dry throat (35.9\%).

The symptomatology and vocal sensations most mentioned by the teachers who reported vocal alterations (GII) were: hoarse voice (56.4\%), voice loss $(41 \%)$, voice failure $(56.4 \%)$, lack of air $(30.8 \%)$, as well as throat burning $(64.1 \%)$, dry cough $(56.4 \%)$, pain while speaking $(48,7 \%)$, vocal difficulty $(47.4 \%)$, fatigue while speaking (33.9\%), pain while swallowing $(30.8 \%)$.

Table 1 shows the comparison of protocol scores between GI and GII.

Table 2 shows the correlations between the scales of the VHI protocol. For VHI, the organic sub-area presented a significantly greater score, both in GI and GII ( $p<0.001)$.

Table 3 shows the comparisons between the domains of V-RQOL. As for V-RQOL, both in GI and GII, the score on the social-emotional sub-domain is greater than that in the physical functionality sub-domain $(p<0.001)$.

Table 4 presents the comparisons among the sections of VAAP. In VAPP, one observes that for GI, the results for the sections dealing with 
Table 1- Comparison of the results of psychometric tests between GI (reported no vocal alteration) and GII (reported vocal alteration)

\begin{tabular}{lccccc}
\hline & Mean & Gd & Mean & Sd & p \\
\hline V-RQOL & & & & & \\
\hline Total & 94.3 & 7.8 & 78.1 & 20.9 & $0.001^{*}$ \\
\hline Physical Functionality & 92.0 & 9.0 & 73.0 & 24.0 & $0.001^{*}$ \\
\hline Social-emotional & 97.7 & 7.7 & 85.3 & 20.5 & $0.001^{*}$ \\
VHI & & & & & \\
\hline Total & 11.7 & 9.6 & 30.7 & 21.6 & $0.001^{*}$ \\
\hline Organic & 6.4 & 4.8 & 15.1 & 8.6 & $0.001^{*}$ \\
\hline Functional & 3.5 & 3.5 & 7.5 & 6.7 & $0.001^{*}$ \\
\hline Emotional & 1.8 & 3.1 & 7.8 & 7.8 & $0.001^{*}$ \\
\hline VAPP & & & & & \\
\hline Total & 14.6 & 21.8 & 55.4 & 53.2 & $0.001^{*}$ \\
\hline Severity & 1.0 & 1.5 & 2.8 & 2.3 & $0.001^{*}$ \\
\hline Total Job Effect & 3.2 & 6.6 & 7.4 & 6.9 & $0.001^{*}$ \\
\hline Limitation & 2.1 & 3.6 & 5.1 & 4.8 & $0.001^{*}$ \\
\hline Restriction & 1.2 & 3.2 & 2.3 & 3.1 & $0.007^{*}$ \\
\hline Total Daily Communication & 5.9 & 8.4 & 23.6 & 24.4 & $0.001^{*}$ \\
\hline Limitation & 3.3 & 4.7 & 13.0 & 13.4 & $0.001^{*}$ \\
\hline Restriction & 2.8 & 4.4 & 10.7 & 11.9 & $0.001^{*}$ \\
\hline Total Social Communication & 1.1 & 1.8 & 6.1 & 7.9 & $0.001^{*}$ \\
\hline Limitation & 0.6 & 1.0 & 3.3 & 4.2 & $0.001^{*}$ \\
\hline Restriction & 0.5 & 0.8 & 2.8 & 4.0 & $0.001^{*}$ \\
\hline Emotion & 3.3 & 6.6 & 15.5 & 16.0 & $0.001^{*}$ \\
\hline Total Vocal Activity Limitation & 6.0 & 8.1 & 21.4 & 21.0 & $0.001^{*}$ \\
\hline Total Vocal Activity Restriction & 4.5 & 7.6 & 15.7 & 18.1 & $0.001^{*}$ \\
\hline V-RQOL Vol & & & & &
\end{tabular}

V-RQOL=Voice-Related Quality of Life

$\mathrm{VHI}=$ Voice Handicap Index

VAPP=Voice Activity Participation Profile

$\mathrm{Sd}=$ Standard deviation

Table 2 - Comparison among the sub-areas of Voice Handicap Index(VHI) for GI (reported no vocal alteration) and GII (reported vocal alteration)

\begin{tabular}{lcccccc}
\hline & VHI & & GI & & & GII \\
& Mean & Median & dp & Mean & Median & SD \\
\hline Organic & $6.4^{\mathrm{a}}$ & 6.0 & 4.8 & $15.1^{\mathrm{a}}$ & 14.0 & 8.6 \\
Functional & $3.5^{\mathrm{b}}$ & 2.0 & 3.5 & $7.5^{\mathrm{b}}$ & 6.0 & 6.7 \\
& $1.8^{\mathrm{c}}$ & 0.0 & 3.1 & $7.8^{\mathrm{b}}$ & 6.0 & 7.8 \\
Emotional & & & & & \\
$\mathrm{P}$ & $<0.001^{*}$ & & & $<0.001^{*}$ & & \\
\hline
\end{tabular}

*statistically significant difference $(p<0.05)$

Sub-areas with different letters, within each group, possess statistically significant differences among themselves.

VHI=Voice Handicap Index

SD=Standard Deviation 
Table 3- Comparison between the domains of Voice-Related Quality of Life (V-RQOL) for groups GI (reported no vocal alteration) and GII (reported vocal alteration)

\begin{tabular}{lcccccc}
\hline \multicolumn{1}{c}{ V-RQOL } & GI & & \multicolumn{2}{c}{ GII } \\
& Mean & Median & Dp & Mean & Median & Dp \\
\hline Physical functionality & 92.0 & 95.8 & 9.0 & 73.0 & 79.2 & 24.0 \\
Socio-emotional & 97.7 & 100.0 & 7.7 & 85.3 & 93.8 & 20.5 \\
$\mathrm{p}$ & $<0.001^{*}$ & & & $0.001^{*}$ & & \\
\hline
\end{tabular}

*statistically significant difference $(p<0.05)$

V-RQOL=Voice-Related Quality of Life

Table 4- Comparison among the sub-areas of Voice Ativity Participation Profile (VAPP) for GI (reported no vocal alteration) and GII (reported vocal alteration)

\begin{tabular}{lcccccc}
\hline \multicolumn{1}{c}{ VAPP } & & GI & & & GII \\
& Mean & Median & SD & Mean & Median & SD \\
\hline Job effect & 8.0 & 1.3 & 16.5 & $18.4 \mathrm{a}$ & 11.3 & 17.3 \\
Effect on Daily Communication & 4.9 & 2.3 & 7.0 & $19.7 \mathrm{a}$ & 10.4 & 20.4 \\
Effect on Social Communication & 2.9 & 1.0 & 4.5 & $15.3 \mathrm{~b}$ & 7.5 & 19.7 \\
Emotion & 4.7 & 1.4 & 9.4 & $22.1 \mathrm{a}$ & 14.7 & 22.9 \\
$\mathrm{P}$ & & $0.116 \mathrm{~ns}$ & & & $0.008^{*}$ & \\
\hline
\end{tabular}

ns - non statistically significant difference

* - statistically significant difference $(p<0.05)$

Sub-areas with different letters, within each group, possess statistically significant difference among themselves.

VAPP=Voice Activity Participation Profile

$\mathrm{SD}=$ Standard Deviation

Table 5- Comparison between Limitation and Restriction of Voice Ativity Participation Profile (VAPP) for GI (reported no vocal alteration) and GII (reported vocal alteration)

\begin{tabular}{lcccccc}
\hline & VAPP & GI & & \multicolumn{2}{c}{ GII } \\
& Mean & Median & SD & Mean & Median & SD \\
\hline Limitation & 6 & 2.8 & 8.1 & 21.4 & 12.3 & 21 \\
Restriction & 4.5 & 1.8 & 7.6 & 15.7 & 9.6 & 18.1 \\
$\mathrm{P}$ & & $<0.001^{*}$ & & & $0.001^{*}$ \\
\hline
\end{tabular}

*Statistically significant difference $(p<0.05)$

VAPP=Voice Activity Participation Profile

$\mathrm{SD}=$ Standard Deviation

emotion, effect on work, and effect on daily communication are significantly greater than the social communication section $(p=0.008)$. However, for GI, the sections showed no statistically significant differences $(p=0.116)$.

Table 5 presents the comparison between the scores of limitation and restriction of activities of protocol VAPP. In both in GI and GII, the score of limitation is greater than that of restriction $(p<0.001)$.

\section{DISCUSSION}

One outstanding characteristic of the sample was the predominance of female teachers, which is consistent with national and international literature ${ }^{3,18}$.

When vocal alteration is reported by the teachers themselves, it is termed, "self-reported vocal alteration" or dysphonia. The prevalence of selfreported vocal alteration in this study (39.8\%) was similar to that found in the study by Simões and Latorre $^{17}$ (2006) (48.7\%).

The most reported symptoms by both GI and 
GII, i.e., hoarseness and voice loss, are similar to those found in Ferreira, et al. ${ }^{4}$ (2007), as well as vocal sensations most reported by the 97 teachers: dry throat and fatigue while speaking. Even the teachers who reported no vocal alteration highlighted symptoms and sensations of voice alteration.

The study by Tavares and Martins ${ }^{20}$ (2008) corroborated that vocal alterations might not be perceived by the teachers. The researchers evaluated teachers who reported and teachers who did not report vocal alterations and found laryngeal alterations such as nodules, polyps, Reinke's edema, atrophy, and leukoplasia, in both groups.

There were significant differences between GI and GII (see Table 1) in all the domains of protocols VHI, V-RQOL, and VAPP. These results indicate the teachers who reported vocal alterations realized that the voice had a negative impact on their daily activities and LQ.

Between GI and GII, a mean of 11.7 and 30.7 respectively is observed for total VHI. The mean of total VHI, for GII, was similar to that of Roy's study ${ }^{15}$, developed with teachers who reported vocal alterations, i.e., 31.58. On the validation of $\mathrm{VHI}^{8}$ scores, up to 33.69 are associated with selfperception of a mild problem.

As seen in Table 2, the organic sub-scale of VHI predominated over the other sub-scales, illustrating that teachers have a feeling of laryngeal discomfort and vocal emission difficulty to a greater or smaller degree; however, those in the group that reported no vocal alteration do not regard laryngeal discomfort and vocal emission difficulty as voice alteration.

In other studies with teachers ${ }^{11,12}$, the organic dimension of VHI also demonstrated higher scores when compared to the other dimensions. The vocal signs/symptoms more easily interpreted by teachers are those that cause physical sensations of significant discomfort ${ }^{13}$. Nevertheless, the teachers are not particularly inclined to interpret these signs as indicators of vocal problems, which show a difference in the way everyday experiences are perceived, felt, interpreted, and meant.

As for $\mathrm{V}-\mathrm{RQOL}$, in the study of $\mathrm{V}-\mathrm{RQOL}$ validation ${ }^{7}$, the mean scores for dysphonic and non-dysphonic patients were, respectively, 53.5 and 98 for total score, 55.9 and 98.9 for the social-emotional domain, and 51.9 and 97.3 for the physical functionality domain. Compared to a population with no vocal complaint, the GII sample presented lower scores; however, these were greater than the mean of scores shown by the dysphonic population.

The negative impact of voice on the LQ of the teachers studied herein seems to be mild, although there are significant differences between GI and
GII. The absolute values shown by the protocols and the score of sub-domains, when interpreted, provided data on the dynamics of the relationship between LQ and voice. It was possible to observe that the physical dimension of individuals seems to influence the LQ of teachers more negatively than functional and emotional issues.

The dimension of physical functionality predominated over the socio-emotional one, both in GI and GII (Table 3). Other studies corroborate with the findings, in which voice has a greater impact on the physical functionality dimension than the social-emotional one ${ }^{1,6,10}$. Physical discomfort, such as the sensation of fatigue while speaking, and difficulty speaking with strong intensity in noisy environments, seems to contribute to the perception of the negative vocal impact on life quality related to voice, more than do the factors related to the socio-emotional component.

According to Jardim, et al. ${ }^{9}$ (2007) there seems to be a hierarchy in the impact of each domain on life quality, similar to that seen in most diseases, where physical functionality affects the psychological aspect that, in turn, affects the social one.

Furthermore, Smith, et al. ${ }^{19}$ (1999) state that life quality and health status are different concepts; however, in the medical literature, they are used as synonyms. For these authors, three great dimensions - mental health, physical functionality and social functioning - influence the perception of life quality and health status. Thus, the mental health/psychological well being dimension is more related to $L Q$, while the physical functionality domain is more associated with the perception of health status.

In the study of Jardim, et al. ${ }^{9}$ (2007), the authors highlight that, despite the high prevalence of vocal fatigue among teachers, the scores of V-RQOL did not reflect the expected influence of these symptoms on life quality related to the voices of the teachers studied. Nevertheless, another psychometric protocol applied to the same population revealed that the teachers are getting sick, psychologically. The authors see a strong relation involving stress, emotion, and voice.

The values found in the VAPP protocol in GII are similar to those presented by teachers in another study $22,69.77$. On the other hand, GI values are similar to the mean found in the control group of Ma and Yiu²2 (2002), 16.9.

The activities limitation and restriction values were greater for teachers in GII than for those in GI. This fact suggests the teachers who reported vocal alteration might face greater problems in regard to limitation of their activities owing to voice problems, and might even restrict their teaching activities away from the classroom.

By comparing the means of VAPP sections 
among themselves (Table 4), it was observed that in GII, the vocal impact on the performance of daily activities is the same as in the session work, daily communication, and emotion, being social communication less affected than the areas above. No significant differences were seen among the sections in GI. These data indicate that voice utilization has a greater negative impact on work, daily communication, and communication of emotions than on the process of socialization and interaction of the teachers with their family members, friends, and colleagues. In GI, it was observed that no session predominated over the other, indicating that, in the group of teachers with no vocal complaint, there is no voice impact.

It can be noticed that the teachers perceived the impact of voice more in terms of limitation of their activities than to the restriction of their work. Even those who reported no vocal alterations realized that their voice might limit their professional performance (Table 5). For the teachers, the important aspect is to have voice. Mild vocal alterations do not seem to restrict their teaching activity. In the present study, most teachers rated their vocal alteration as mild and, among these, only $18.4 \%$ reported to have restricted their professional performance because of voice problems. Probably, this professional limitation noted by the teachers, both in GI and GII, is related to the symptomatology and sensations of vocal alterations, as reported by the teachers.

Vocal health programs are important to guide teachers in the suitable use of their voices, in order to diminish the symptomatology and sensations of vocal alterations and laryngeal discomfort reported by these professionals.

Furthermore, health promotion actions may provide the teachers with skills such as attention, perception, and recognition of their own voices in terms of quality, alterations, and transformations, in different social relations and everyday contexts ${ }^{13}$.

\section{CONCLUSIONS}

The teachers with vocal complaints perceived more negative impact on their LQ and their activities in all domains of the protocol, than did the teachers who reported no vocal alteration.

The organic dimension of VHI predominated over the other dimensions in the two groups researched, and the physical functionality domain of $\mathrm{V}-\mathrm{RQOL}$ presented smaller numeric measurements in relation to the social-emotional domain.

The teachers of the two groups realized that a voice alteration limits their professional performance more than it impairs the exercise of their profession.

\section{REFERENCES}

1- Behlau M, Hogykian N, Gasparini G. Quality of life and voice: study of a Brazilian population using the voice-related quality of life measure. Folia Phoniatr Logop. 2007;59:286-9.

2- Behlau M, Oliveira G, Santos LMA, Ricarte A. Validation in Brazil of self-assessment protocols of dysphonia impact. Pro Fono. 2009;21(4):326-32.

3- Delcor NS, Araújo TM, Reis EJFB, Porto LA, Carvalho FM, Silva $\mathrm{MO}$, et al. Labor and health conditions of private school teachers in Vitória da Conquista, Bahia, Brazil. Cad Saúde Públ. 2004;20(1):187-96.

4- Ferreira LP, Giannini SPP, Latorre MRDO, Zenari MS. Distúrbio de voz relacionado ao trabalho: proposta de um instrumento para avaliação de professores. Disturb Comun. 2007;19(1):127-36.

5- Fleck MPA. O instrumento de avaliação de qualidade de vida da Organização Mundial da Saúde (WHOQOL-100): características e perspectivas. Ciên Saúde Coletiva. 2000;5(1):3-38.

6- Gasparini G, Behlau M. Quality of life: validation of the Brazilian version of the voice-related quality of life ( $V$-RQOL) measure. J Voice. $2009 ; 23(1): 76-81$.

7- Hogykian ND, Sethuraman D. Validation of an instrument to measure voice-related quality of life (V-RQOL). J Voice. 1999;13(4):557-69.

8- Jacobson BH, Johnson A, Grywalski C, SIlbergleit A, Jacobson G, Benninger MS, et al. The voice handicap index: development and validation. Am J Speech Lang Pathol. 1997;6(3):66-70.

9- Jardim R, Barreto SM, Assunção AA. Condições de trabalho, qualidade de vida e disfonia entre docentes. Cad Saúde Públ. 2007;23(10):2439-61.

10- Kasama ST; Brasolotto AG. Vocal perception and life quality. Pro Fono. 2007;19(1):19-28.

11- Kooijman PGC, De Jong FI, Oudes MJ, Huinck W, Van Acht $\mathrm{H}$, Graamans K. Muscular tension and body posture in relation to voice handicap and voice quality in teachers with persistent voice complaints. Folia Phoniatr Logop. 2005;57(3):134-47.

12- Kooijman PGC, Thomas G, Graamans K, De Jong FI. Psychosocial impact of the teachers' voice through the career. J Voice. 2007;21(3):316-24.

13- Penteado RZ, Pereira IMTB. Qualidade de vida e saúde vocal de professores. Rev Saúde Públ. 2007;41(2):236-43.

14- Preciado-López J, Pérez-Fernández C, Calzada-Uriondo $M$, Preciado-Ruiz P. Epidemiological study of voice disorders among teaching professionals of La Rioja, Spain. J Voice. 2008;22(4):489508.

15- Roy N, Merril RM, Thibeault S, Parsa RA, Gray S, Smith EM. Prevalence of voice disorders in teachers and the general population. J Speech Lang Hear Res. 2004;47:281-93.

16- Seidl EMF, Zannon CMLC. Qualidade de vida e saúde: aspectos conceituais e metodológicos. Cad Saúde Públ. 2004;20:580-9.

17- Simões M, Latorre MRDO. Prevalência de alteração vocal em educadoras e sua relação com a auto-percepção. Rev Saúde Públ. 2006;40(6):1013-8.

18- Smith E, Gray SD, Dove H, Kirchner L, Heras H. Frequency and effects of teacher's voice problems. J Voice. 1997;11(1):81-7. 19- Smith KW, Avis NE, Assman SF. Distinguishing between quality of life and health status in quality of life research: a meta-analysis. Qual Life Res. 1999;8(5):447-59.

20- Tavares ELM, Martins RHG. Vocal evaluation in teachers with or without symptoms. J Voice. 2008;21(4):407-14.

21- Verdolini K, Raming LO. Review: occupational risks for voice problems. Logoped Phoniatr Vocol. 2001;26(1):37-46.

22- Yiu EML, Ma EPM. Voice activity limitation and participation restriction in the teaching professing: the need for preventive voice care. J Med Speech Lang Pathol [serial on the internet]. 2002 [cited 2011 Aug 24th]; 1,Mar. Available from: http://findarticles. com/p/articles/mi_hb1397/is_1_10/ai_n28956173/. 